\title{
Selection of Laboratory Mice for the High Scores of Logic Task Solutions: The Correlated Changes in Behavior
}

\author{
Olga V. Perepelkina, Vassilissa A. Golibrodo, Irina G. Lilp, Inga I. Poletaeva \\ Laboratory of Physiology and Genetics of Behavior, Biology Faculty, Lomonossov Moscow State University, \\ Moscow, Russia \\ Email: ingapoletaeva@mail.ru
}

Received 30 December 2013; revised 3 February 2014; accepted 20 February 2014

Copyright @ 2014 by authors and Scientific Research Publishing Inc.

This work is licensed under the Creative Commons Attribution International License (CC BY). http://creativecommons.org/licenses/by/4.0/

(c) (i) Open Access

\begin{abstract}
Genetically heterogenous mouse population served as the basis for experiment designed to select mice for the high levels of extrapolation ability. Extrapolation task (which belongs to the category of cognitive tasks) was the following: hungry and thirsty mouse was placed in the experimental box facing the cup with milk which could be reached via the small opening in the frontal wall. After several seconds of drinking the food moved behind this wall to the right or to the left and disappeared from animal view. Mouse can find the food only if it moves in the direction of food movement. The direction could be chosen at random or based on animal apprehension of elementary logic of this task. Mice were selected not only for high scores of this task solution but also for lack of anxiety signs during test performance. Extrapolation task scores in selected strain mice were above the chance levels in F9 but non-significantly different from those of control unselected mouse population. The performance of F9 mice in the "puzzle box" cognitive test, based on animal tendency to avoid the brightly lit area in the dark compartment with increasing "difficulty" of reaching this compartment, was measured as mean escape latencies of the respective group. The latencies of mice from selected strain were significantly shorter than those of control animals. This fact is interpreted as the sign that the selection for one cognitive trait (yet still not efficient) resulted in positive changes in the ability to solve another cognitive task. The relationship of cognitive task solutions success and anxiety behavior scores in F9 is discussed.
\end{abstract}

\section{Keywords}

Animal Cognition; Genetic Selection; Extrapolation Capacity; Puzzle Box; Anxiety; Laboratory Mice 


\section{Introduction}

Animal reasoning ability (e.g. ability of an animal to apprehend the connections between objects and events in the environment) is the continuum of phenomena with mechanisms different from those of classical and instrumental conditioning [1]. The genetic component in the variability of learning capacities was proven in bothclassical genetic studies (selection experiments, mutations, interstrain differences) and in numerous studies with gene expression manipulations. The genetic control of cognitive traits (radial maze and Morris water maze performances) was also proven both as the existence of interstrain differences in rats and mice and in mice with knockouts (and knock-ins) of numerous genes, which participate in signaling cascades involved in plasticity of neuronal functions. At the same time, there were no selection experiments with selection for high scores of respective traits.

L. V. Krushinsky, the prominent Russian neurobiologist, introduced into the scientific practice the notion of animal reasoning. He and his colleagues performed experiments in which this ability was evaluated quantitatively [2]. The interspecies comparisons in experiments from Krushinsky’s laboratory demonstrated the positive correlations between animal ability for extrapolation and the degree of brain complexity. The extrapolation capacity test implies that animal is able (or unable) to find the stimulus (usually food bait) which is initially available (via the narrow gap in the screen), but then starts movement to the left or to the right, disappearing from animal view behind the opaque screen. Animal can follow this movement for the certain distance (in the case of mouse experiment it is $1 \mathrm{~cm}$ ). After the stimulus disappears from view animal can found it behind the screen if it moves in the respective direction. Thus the positive task solution is the approach to the food bait in its new position. Animal has to understand that the object, which disappears during this movement, still exists, which means that animal is able to operate the rule of "object permanence” according to J. Piaget [3]. The ability to extrapolate the direction of stimulus movement on the invisible part of its trajectory is another capacity which animal has to possess in order to be able to solve this task. The task success solution is estimated by the proportion of correct choices made by a given animal or a group of animals. It is obvious that the task solution during the first presentation is the most important score of this test as an animal, performing the extrapolation task for the first time, has no analogous previous experience and thus behaves according to its reasoning capacity. At the same time, in most experiments the task was presented for 6 times. Thus two scores were characteristic for the animal group-the proportion of correct solutions performed by this group at first task presentation and the similar score for their summed success for 1 - 6 task presentations.

It was shown that rodents (rats and mice) of laboratory strains have the low capacity for extrapolation, while wild Norway rats and F1-2 hybrids of Norway rat x laboratory rats performed much more successfully. This fact was the indication of the plausible genetic bases of this ability and the attempt to select rats of this hybrid population for high extrapolation performance was performed in Krushinsky laboratory [4]. This selection experiment failed, as rats of F3-F-4 selection generations demonstrated the high level of anxiety behavior in the experimental box which made it impossible to test their extrapolation ability. Experiments with mice demonstrated that certain genetic groups (i.e. carrying robertsonian translocation-fusion of chromosomes nn. 8 and 17) were capable to solve the extrapolation task in statistically significant proportion of cases [5]. Thus while planning the new selection experiment in attempt to select mice for high extrapolation ability it was decided to select animals for two traits at a time-for high score of extrapolation task solution and for low anxiety signs during this test performance. Such signs of anxiety were-the chaotic "nervous" locomotion of an animal when it was placed in the experimental box, "the refusals" to approach food in the experimental situation and the "zero" solutions when animal did not approach the food (or the wrong place, where food was absent) during 120 sec. During the course of this experiment the response to selection for lower level of anxiety was evident-the significant decrease of "refusals" and "zero" solutions in selection generation in the selected strain (EX) took place in comparison to mice of non-selected control population (CoEX). The success of extrapolation task solutions could be estimated by the proportion of "correct" task solution which is significantly above the $50 \%$ level which is characteristic for the chance level of performance. The significant prevalence of correct choices in EX mice in F3 F6 and in F8 was evident, although the performance of CoEx mice occasionally was significantly above the chance level as well [6]. The differences between the proportions of successful extrapolation task solutions between EX and CoEX mice in selection generations were either non-significant or absent. It means that there was no overt "response to selection" for this trait. Thus the goal of this paper was to analyze the behavior of F9 EX mice not only in extrapolation test but in other tests as well, comparing their performance to that of control ani- 
mals. These tests were "puzzle box test [7] and elevated plus maze (EPM) test.

\section{Methods and Material}

Animals. The selection of mice for high extrapolation ability was started on the basis of genetically heterogenic population (they were F4 hybrids of crosses between strains selected for large and small relative brain weight (descending from 6 inbred straions) [6]. The criteria for selecting animals as parents for the next generation were: correct task solution at the first extrapolation task presentation, the 5 - 6 correct solution from 6 task presentations, lack of "hesitation" in approaching food (no refusals), no "zero" solutions (when no choice was made during $120 \mathrm{sec}$ ). Mice of control (CoEx) population were bred at random.

Behavioral tests. Extrapolation test. The experimental device for testing the extrapolation ability was the opaque open box which contained two reward chambers and the central feeding site. At these locations the mouse could reach a small food cup containing milk through holes (diam. $10 \mathrm{~mm}$ ). Two identical cups were mounted on the bar in front of the wall (outside the box) and could be slid manually to the lateral feeding sites. One of these cups could be moved to the right, another-to the left from central feeding site. Mice were food and water derived for 15 - 16 hours. On the test day individual mouse was placed into the box. Animal started to drink milk from one of the cups via the central opening. After 3 - 5 sec of drinking the cup was moved slowly to the right or to the left. The mouse could follow this displacement for $1-2 \mathrm{~cm}$ of the trajectory and then the food cup disappears from its view. The second cup (also containing milk) was moved in the opposite direction staying invisible for an animal (this was performed to balance the odor cues from both sides of the box). The cup from which animal started to drink moved to the respective side opening. The choice of the feeding location (either indicated by perceived movement of the food cup or by chance) was registered as the correct task solution while the cases when animal approached to the opposite side opening was qualified as incorrect solution. If no approach was performed for $120 \mathrm{sec}$ it was qualified as "zero" solution (see above). The experimental session included six trials. The data were presented as the two separate scores-proportions of correct task solutions from the total number of them both for the first task presentation and for six presentations in sum. 107 mice of EX strain (males and females) and 88 mice of CoEX population from F9 were tested for extrapolation ability.

"Puzzle box" test, described in details by Ben-Abdallah et al. [8], is the modified Light-Dark box, in which the route to the dark compartment (goal box) was via an underpass ( $4 \mathrm{~cm}$ wide, $2 \mathrm{~cm}$ deep and $15 \mathrm{~cm}$ long). This entrance could be blocked by either wood shavings (burrowing puzzle) or by a T-shaped card-board-plastic plug (plug puzzle). There were 10 stages of the test performed during 3 experimental days. The test started by the simple stages 1 - 4 (animal can enter the dark part of the box), the next stages (5 - 7) possessed the "cognitive" component, when the underpass was filled by wood shavings to the level of the box floor, then followed stages 8 - 9 (in which the underpass was blocked by low weight plug, which mouse could easily lift and put aside in order to penetrate the goal box) and stage 10, in which the wood shaving heap (5 - $7 \mathrm{~cm}$ high) was placed along the all wall with the underpass (for details see Ben-Abdallah et al., 2012). "Cognitive” component of this task solution was based on the fact that animal should comprehend that if the entrance to the goal box was not seen it still existed. Thus animal have to understand the "object permanence" rule. The group mean time scores for a group of animals (latencies to enter the goal box) were used to measure the task solution success. 41 EX mice and 39 CoEX mice were tested in puzzle box. One half of each group was tested before extrapolation and EPM tests, another half-after these tests.

Elevated plus maze $(E P M)$ was the standard device, placed $28 \mathrm{~cm}$ above the floor, arm length-20 cm, open arms width $-4.7 \mathrm{~cm}$, closed arms width $-5.1 \mathrm{~cm}$, walls $14.2 \mathrm{~cm}$ high, the central area $-4.7 \times 5.1-4.7 \times 5.1 \mathrm{~cm}$. Mouse was placed onto the central platform and allowed to explore the apparatus for 3 min. The behavioral acts evaluated included two traditional variables_time spent in the open-arms and the number of open-arm entriesrisk assessment activities_- "head dips" (scanning over the sides of the maze towards the floor), numbers of rearings, grooming episodes and defecation boli. 26 EX mice and 65 CoEX F9 mice were tested in EPM.

Statistics. The significance of the proportions above the chance level was determined using Fisher $\varphi$-method. The statistical significance of inter strain differences in puzzle box solution latencies and in EPM variables was assessed by two or one factor ANOVA with post-hoc LSD Fisher test evaluation.

\section{Results}

Extrapolation task success in F9 EX and CoEX mice. The proportions of mice which solved the extrapolation 
task successfully is presented in Table 1.

The data indicated the slight prevalence of EX mice performance in extrapolation task (statistically non-significant). Note, that these animals belonged to $9^{\text {th }}$ generation of breeding the best extrapolation performers of the EX strain and still no difference between EX and control mice were detected. Extrapolation scores demonstrated the small prevalence of correct choices in both cohorts (EX and CoE-X) of female mice in comparison to respective male groups (data not presented).

"Puzzle box" test. The results of "puzzle box" test are shown in Figure 1.

The performance of animals in this test is based on fear-anxiety motivation (while extrapolation test performance is possible using food motivation). Thus the solution success in this test could be influenced by handling experience, which ameliores anxiety [9] [10]. EX and CoEX mice, tested in this study, received the moderate handling treatments, during previously applied extrapolation test and in EPM. In order to control handling effect variable two groups of EX and CoEX mice were tested before and after the extrapolation and EPM tests respectively (Figure 1).

Comparison of these two pairs of curves revealed several subtle differences which were although not statistically significant. Thus the performance of EX and CoEX mice in "puzzle box" test did not depend on plausible differences in anxiety induced by handling and reflect differences in their capacities as plausible changes in cognitive ability which resulted from selection for the extrapolation capacity.

Elevated plus maze test. As mentioned above, the selection program adopted in this study implied the selection for high extrapolation scores and for lack of anxiety signs during the procedure of this test. In earlier selection generations the response to selection for latter trait was evident (see Figure 2).

EX mice demonstrated the significantly higher tendency for open arms exploration (time in open arms, number of open arm visits and head drippings) and less numbers of grooming episodes in comparison to CoEX mice

In F9 mice the gender differences in EPM scores were found. The time spent in open arms, numbers of visits to open arms and of head dips as well as of grooming episodes were significantly $(\mathrm{p}<0.001)$ higher in EX females in comparison to their males, with no gender differences being found in CoEX mice (data not shown). The gender differences with non-significantly higher scores in F9 EX female mice were mentioned above.

\section{Discussion}

The term “animal cognition” is usually vaguely defined by authors [1] and assumes to include phenomena which

Table 1. The proportions of correct solutions of extrapolation task solutions for F9 EX and CoEX mice.

\begin{tabular}{ccccc}
\hline Group, number of subj & $\begin{array}{c}\text { Success of } 1^{\text {st }} \text { extrfpolation } \\
\text { task solution (in\%) }\end{array}$ & $\mathrm{p}^{*}$ & $\begin{array}{c}\text { Success of } 6 \text { extrapolation. } \\
\text { tasks solution }\end{array}$ & $\mathrm{p}^{*}$ \\
\hline $\mathrm{EX} \mathrm{n}=107$ & 68.2 & $\mathrm{p}<0.001$ & 70.7 & $\mathrm{p}<0.001$ \\
$\mathrm{CoEX} \mathrm{n}=88$ & 62 & $\mathrm{p}<0.01$ & $59 \%$ & $\mathrm{p}<0.001$ \\
\hline
\end{tabular}

*significantly different from $50 \%$ chance level of choices.
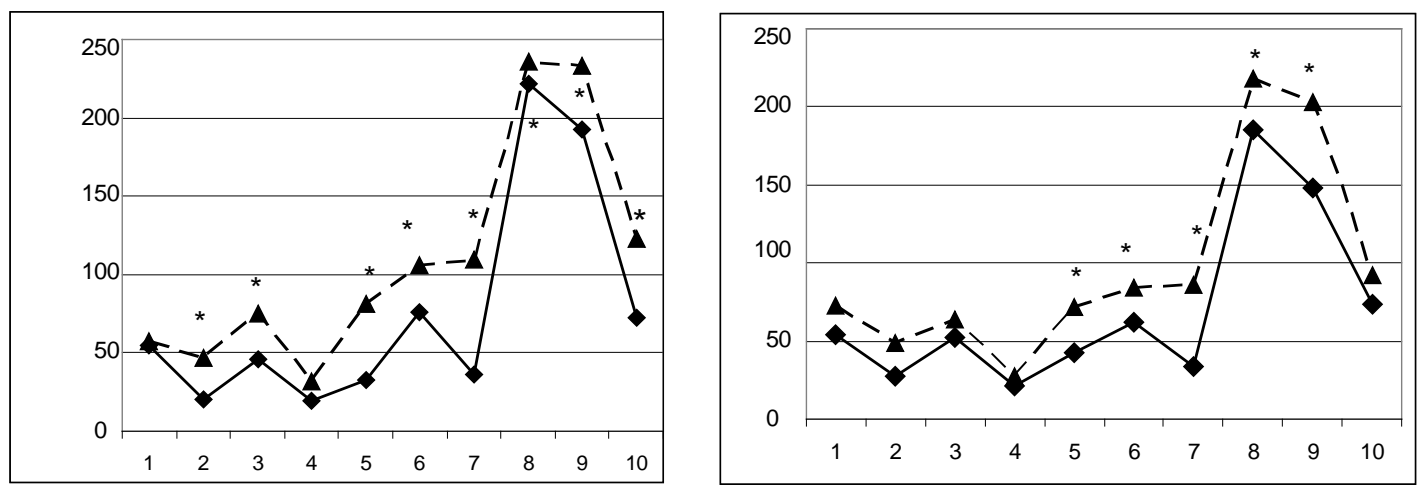

Figure 1. The latencies (ordinate, sec) of "puzzle box” test solutions by F9 Ex and CoEX mice during successive test stages (abcissae). Left graph: experiment, performed before the extrapolation and EPM tests, right: when puzzle box was applied after these tests. Rhombs: performance of EX strain, triangles: CoEX population. * sign. different from EX strain values, $\mathrm{p}<0.05$. 

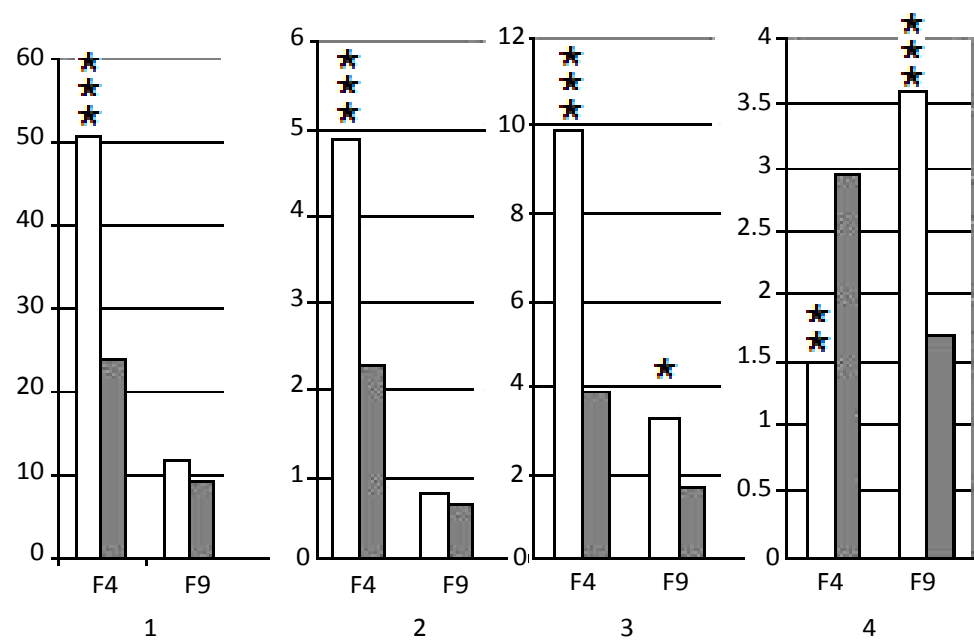

Figure 2. Elevated plus maze scores in EX and CoEX mice from F4 and F9 generations (intergroup differences evaluated by 1 factor ANOVA). EXwhite bars, CoEX - grey bars. 1: time, spent in open arms; 2: open arm visits; 3: head dips from open arms; 4: number of grooming episodes (in closed arms); ${ }^{*}{ }^{* *},{ }^{* * *}$ sign. different from respective CoEX scores.

range from habituation and attention to human speech and its analogues in apes. The ability of animals for spatial learning belongs to the category of cognitive phenomena per se, as they are not based on stimulus-reaction principle of conditioning but involve more complicated mechanisms of spatial map formation. The extrapolation task solution also requires the capacity of elementary logic task solution (as well as the "puzzle box" paradigm). But no experimental attempts were made to select strain of rats (or mice) for high and low ability to learn spatial tasks. At the same time numerous experimental works proved the existence of genetic differences either among laboratory rodent strains or in animals with artificially changed genotype [11]-[13]. This means that our selection experiment is the only one in which the complicated cognitive trait is selected for high performance values. As mentioned above laboratory mice of inbred strains display low capacity for extrapolation [5]. At the same time mice which had the fused $8^{\text {th }}$ and $17^{\text {th }}$ chromosomes (robertsonian translocation) solved this task successfully. This was shown both in mice in which fused chromosomes were transferred to different genetic backgrounds (C57BL/6J and $\mathrm{CDF} / \mathrm{Lac} / \mathrm{Sto}$ ) and in mice, in which the translocation of these chromosomes originated from geographically different wild mouse populations [5].

Learning ability (which is also the cognitive trait) demonstrated inter strain differences as well [14] [15] and successful selection experiments were performed, the Roman High and Low Avoidance rat strains being the most well known example of such selection [16].

Analysis of behavior in F9 mice which were selected for high extrapolation ability was disappointing as no increase in the correct task solutions proportion was achieved. But the results of "puzzle box" test presentation demonstrated, that EX mice solved this task significantly quicker than mice from control population. It means that the selection process did induce some changes in mouse ability to solve the task, although the relationships of genetic and environmental factors affecting this behavior are not at all simple.

The "coordination" of anxiety and cognitive traits in EX mice was also rather difficult to interpret. The lower anxiety (EPM test) in EX F4 mice looked rather "natural" as our experiment implied the selection against elevated anxiety. The decreased anxiety was seen more conspicuously in EPM test (although mice were selected against anxiety signs during extrapolation test). Although in F9 this difference was much less accentuated—both open arm time and numbers of open arm visits were nonsignificantly different from scores of controls. The effects of selection for nonanxiety trait (wheel running) on EPM anxiety scores was also found in the experiment, described by Jonas et al. [17]. At the same time, authors reported that mice of this strain were more investigative in the open field test, demonstrating both increased locomotion in the center of the arena and the increased rearing activity The increase of anxiety behavior in EPM was found in one of their mouse strains [17], and the increased rearings and locomotion in the center of the open field as well as higher number of approaches to a novel object were also noted. These data and our result have the feature in common —-they demonstrate that the rela- 
tionship of "cognition-related" behavior and anxiety behavior is rather complicated. The probable explanations of this seemingly contradictory data could be in the complexity of mechanisms, which are used by a mouse brain for cognitive task solution (and the complexity of its genetic basis as well). The non-uniform expression of state anxiety of mice in different experimental contexts could be also the cause of such complex relationships between scores from different tests. The first suggestion (the complex nature of both-anxiety and cognitive behavior) is indirectly supported by numerous data in which the modulation of animal cognition is achieved by genetic manipulation of numerous genes which belong to different signaling cascades and express in different brain areas [18] thus activating them differentially. The second suggestion is supported by several findings, in which the nonuniformity of anxiety traits was demonstrated [19]. Phenotypically similar anxiety traits, observed in different contexts, could be under the control of different neurotransmitter systems [20]. The behavior of EX and CoEX mice of next generations will probably elucidate this issue.

\section{Acknowledgements}

Supported by grants from RFBR (NN 10-04-00891 and 13-04-00747).

\section{References}

[1] Reznikova, Z. (2007) Animal Intelligence. From Individual to Social Cognition. Cambridge University Press, Cambridge.

[2] Krushinsky, L.V. (1990) Experimental Studies of Elementary Reasoning. Evolutionary, Physiological and Genetic Aspects of Behavior, Oxonian Press, New Dehli.

[3] Zucca, P., Milos, N. and Vallortigara, G. (2007) Piagetian Object Permanence and Its Development in Eurasian Jays (Garrulus glandarius). Animal Cognition, 10, 243-258. http://dx.doi.org/10.1007/s10071-006-0063-2

[4] Krushinsky, L.V., Astaurova, N.V., Kouznetzova, L.V., Otchinskaya, E.I., Poletaeva, I.I., Romanova, L. G. and Sotskaya, M.N. (1975) The Role of Genetic Factors in Determining the Extrapolation Ability in Animals. In: Fedorov, V.K. and Ponomarenko, V.V., Eds., Current Problems in Behavioural Genetics, Nauka, Leningrad, 98-110.

[5] Poletaeva, I.I., Romanova, L.G. and Popova, N.V. (1993) Genetic Aspects of Animal Reasoning. Behavior Genetics, 23, 467-475. http://dx.doi.org/10.1007/BF01067982

[6] Perepelkina, O.V., Markina, N.V., Golibrodo, V.A., Lil’p, I.G. and Poletaeva, I.I. (2011) Selection of Mice for High Level of Extrapolation Capacity with Cobcommitant Low Anxiety Level. Zhurnal Vysshei Nervnoi Deyatelnosti Imeni I P Pavlova, 61, 742-749.

[7] Galsworthy, M.J., Paya-Cano, J.L., Liu, L., Monleón, S., Gregoryan, G., Fernandes, C., Schalkwyk, L.C. and Plomin, R. (2005) Assessing Reliability, Heritability and General Cognitive Ability in a Battery of Cognitive Tasks for Laboratory Mice. Behavior Genetics, 35, 675-692. http://dx.doi.org/10.1007/s10519-005-3423-9

[8] Ben Abdallah, N.M., Fuss, J., Trusel, M., Galsworthy, M.J., Bobsin, K., Colacicco, G., Deacon, R.M., Riva, M.A., Kellendonk, C., Sprengel, R., Lipp, H.-P. and Gass, P. (2011) The Puzzle Box as a Simple and Efficient Behavioral Test for Exploring Impairments of General Cognition and Executive Functions in Mouse Models of Schizophrenia. Experimental Neurology, 227, 42-52. http://dx.doi.org/10.1016/j.expneurol.2010.09.008

[9] Andrews, N. and File, S.E. (1993) Handling History of Rats Modifies Behavioural Effects of Drugs in the Elevated Plus-Maze Test of Anxiety. European Journal of Pharmacology, 235, 109-112. http://dx.doi.org/10.1016/0014-2999(93)90827-5

[10] Costa, R., Tamascia, M.L., Nogueira, M.D., Casarini, D.E. and Marcondes, F.K. (2012) Handling of Adolescent Rats Improves Learning and Memory and Decreases Anxiety. Journal of the American Association for Laboratory Animal Science, 51, 548-553.

[11] Zilles, K., Wu, J., Crusio, W.E. and Schwegler, H. (2000) Water Maze and Radial Maze Learning and the Density of Binding Sites of Glutamate, GABA, and Serotonin Receptors in the Hippocampus of Inbred Mouse Strains. Hippocampus, 10, 213-225. http://dx.doi.org/10.1002/1098-1063(2000)10:3<213::AID-HIPO2>3.0.CO;2-Q

[12] Steinberger, D., Reynolds, D.S., Ferris, P., Lincoln, R., Datta, S., Stanley, J., Paterson, A., Dawson, G.R. and Flint, J. (2003) Genetic Mapping of Variation in Spatial Learning in the Mouse. The Journal of Neuroscience, 23, 2426-2434.

[13] Brooks, S.P., Pask, T., Jones, L. and Dunnett, S.B. (2005) Behavioural Profiles of Inbred Mouse Strains Used as Transgenic Backgrounds. II: Cognitive Tests. Genes, Brain and Behavior, 4, 307-317. http://dx.doi.org/10.1111/j.1601-183X.2004.00109.x

[14] Podhorna, J. and Brown, R.E. (2002) Strain Differences in Activity and Emotionality Do Not Account for Differences in Learning and Memory Performance between C57BL/6 and DBA/2 Mice. Genes, Brain and Behavior, 1, 96-110. 
http://dx.doi.org/10.1034/j.1601-183X.2002.10205.x

[15] Zarcone, T.J., Chen, R. and Fowler, S.C. (2004) Differential Acquisition of Food-Reinforced Disk Pressing by CD-1, BALB/cJ and C57BL/6J Mice. Behavioural Brain Research, 52, 1-9.

[16] Escorihuela, R.M., Fernandez-Teruel, A., Gil, L., Aguilar, R., Tobena, A. and Driscoll, P. (1999) Inbred Roman Highand Low-Avoidance Rats: Differences in Anxiety Novelty-Seeking, and Shuttlebox Behaviors. Physiology \& Behavior, 67, 19-26. http://dx.doi.org/10.1016/S0031-9384(99)00064-5

[17] Jónás, I., Schubert, K.A.A., Reijne, C., Scholte, J., Garland, T., Gerkema, M.P., Scheurink, A.J.W., Nyakas, C. and van Dijk, G. (2010) Behavioral Traits Are Affected by Selective Breeding for Increased Wheel-Running Behavior in Mice. Behavior Genetics, 40, 542-550. http://dx.doi.org/10.1007/s10519-010-9359-8

[18] Paratore, S., Alessi, E., Coffa, S., Torrisi, A., Mastrobuono, F. and Cavallaro, S. (2006) Early Genomics of Learning and Memory: A Review. Genes, Brain and Behavior, 5, 209-221.

[19] Gomes, V. de C., Hassan, W., Maisonnette, S., Johnson, L.R., Ramos, A. and Landeira-Fernandez, J. (2013) Behavioral Evaluation of Eight Rat Lines Selected for High and Low Anxiety-Related Responses. Behavioural Brain Research, 257, 39-48. http://dx.doi.org/10.1016/j.bbr.2013.09.028

[20] Wierońska, J.M., Smiałowska, M., Brański, P., Gasparini, F., Kłodzińska, A., Szewczyk, B., Pałucha, A., ChojnackaWójcik, E. and Pilc A. (2004) In the Amygdala Anxiolytic Action of mGlu5 Receptors Antagonist MPEP Involves Neuropeptide Y but Not GABAA Signaling. Neuropsychopharmacology, 29, 514-521.

http://dx.doi.org/10.1038/sj.npp.1300322 